\title{
Estudio de validez de contenido y confiabilidad de un instrumento para diagnosticar el proceso de inclusión en las Escuelas Normales
}

\section{A Study of Content Validity and Reliability of an Instrument to Diagnose Inclusion Processes in Teacher Colleges}

DOI: https://doi.org/10.32870/dse.v0i22.698

Hernán Hernández Ayala*

Sergio Tobón Tobón**

Luis Gibran Juárez-Hernández***

\begin{abstract}
Resumen
Se requiere contar con instrumentos para evaluar los procesos de inclusión en las escuelas normales que sean pertinentes a los retos de la sociedad del conocimiento y que apliquen la socioformación. En este sentido, el objetivo del presente estudio fue determinar la validez y confiabilidad de una rúbrica para diagnosticar procesos de inclusión en las escuelas normales, acorde con la socioformación, para promover una mejor toma de decisiones en la educación normal. Para ello, se realizó un estudio instrumental con base en los siguientes pasos: 1) contrastación de la validez de constructo mediante la técnica de Análisis Factorial; y 2) determinación de la confiabilidad del instrumento mediante la aplicación a una muestra de 433 participantes. Los resultados del análisis factorial cumplieron con los requisitos para el análisis factorial exploratorio (EFA); se obtuvieron correlaciones estadísticamente significativas entre los ítems de la rúbrica, los valores de las comunalidades adecuados y el EFA extrajo un componente principal; el análisis de confiabilidad muestra un valor de Alfa de Cronbach de 0.915. Como conclusión, se presenta un instrumento pertinente para diagnosticar procesos de inclusión en la educación orientada a la formación de profesores, en virtud de que, al ser evaluado el instrumento, mostró una concordancia significativa en su contenido y una alta confiabilidad.
\end{abstract}

Palabras clave: educación - formación de docentes - juicio de valor - evaluación - sociedad del conocimiento.

\section{Abstract}

It is necessary to have instruments to evaluate inclusion processes in teacher colleges that are pertinent to the challenges of an information society and that apply socially-oriented training. In this respect, the aim of this study was to determine the validity and reliability of a rubric to diagnose inclusion processes

\footnotetext{
* Maestro en Educación y doctorante en el Centro Universitario CIFE-Universidad Pedagógica Nacional. Profesor de la Universidad Autónoma de Sinaloa y la Escuela Normal Experimental de El Fuerte, Sinaloa, México. heah749@hotmail.com

** Doctor en Modelos Educativos y Políticas Culturales. Director General e Investigador del Centro Universitario CIFE, Cuernavaca, Morelos, México. stobon@cife.ws

*** Doctor en Ciencias Biológicas y de Salud con especialidad en Ecología. Profesor-investigador del Centro Universitario CIFE. Cuernavaca, Morelos, México. luisgibrancife@gmail.com
} 
in teacher colleges in accordance with a social orientation to promote better decision-making in teacher education. To do this, an instrumental study was carried out based on the following steps: 1) contrasting the construct's validity using the Factor Analysis technique, and 2) determining the reliability of the instrument by applying it to a sample of 433 participants. The results of the factor analysis met the requirements for exploratory factor analysis (EFA). Statistically significant correlations were obtained between the items of the rubric, adequate commonalities values and the EFA extracted a main component. The reliability analysis shows a Cronbach's Alpha value of 0.915. As a conclusion, a pertinent instrument is presented to diagnose inclusion processes in education aimed at teacher training, since, when evaluated, it showed a significant concordance in its content and high reliability.

Keywords: education - teacher training - value judgment - evaluation - information society.

\section{Introducción}

La educación inclusiva es una tendencia educativa y social que en los últimos años ha contribuido a hacer conciencia de las necesidades de trasformación respecto a condiciones culturales, políticas y de prácticas favorecedoras en los procesos de inclusión. Según Latorre (2013), las instituciones educativas $y$, particularmente, las instituciones formadoras de docentes, no pueden ser ajenas a esta situación y deben tomar medidas en la formación de docentes, concebida como uno de los factores decisivos del sistema educativo y de la sociedad. En este sentido, se reconoce que desde hace algunos años, en México se promueve la educación inclusiva; como muestra de ello, se concreta el término de inclusión a partir de la Reforma Integral de Educación Básica en el Acuerdo 592, donde se refiere a la inclusión como un medio para favorecer la atención a la diversidad, reconociendo que en las escuelas la diversidad se manifiesta en la variedad lingüística, social, cultural, de capacidad, ritmos y estilos de aprendizaje (SEP, 2011). Por lo tanto, si el Sistema Educativo Nacional pretende alcanzar estándares de calidad y equidad, debe promover el desarrollo de procesos de inclusión en las escuelas, particularmente en aquellas dedicadas a la formación de profesores, cuya educación debe responder a la trasformación social, cultural, científica y tecnológica que se vive en nuestro país y en el mundo (SEP, 2012).

Para la escuela, la inclusión representa una búsqueda constante de mejoras e innovaciones en su filosofía, práctica y organización que le permita optimizar la presencia, la participación y el éxito de todos los estudiantes al responder positivamente a la diversidad del alumnado, en donde las diferencias se asuman como un estímulo para fomentar el aprendizaje entre todos (García, 2015). Por tanto, se debe postular una concepción de institución educativa que tenga apertura a la diversidad, capaz de atender y dar respuesta a las necesidades de todos los estudiantes. Reconociendo que tales necesidades se han incrementado debido a los cambios sociales, como el aumento de la migración de la población, el reconocimiento de la riqueza cultural y lingüística, y los derechos de las comunidades, entre otros. Es así como la preocupación por la

Diálo@os sobre Educación año 12 | número 22 | enero-junio 2021 | ISSN 2007-2171 
escuela inclusiva se ha convertido en uno de los mayores retos que actualmente deben afrontar los sistemas educativos, los centros, el profesorado y la sociedad (Durán, Climent, 2017).

Es preciso señalar que avanzar hacia una escuela inclusiva es un proceso complejo; para hacer frente a este reto es importante realizar investigaciones en torno a los procesos que requiere una escuela para convertirse en un espacio inclusivo. Para ello, se deben retomar los aportes de todos los actores que participan en los procesos de formación que potencian sus posibilidades, sistematizar las experiencias y aprender de la voz de quienes protagonizan los procesos de inclusión. Considerando con esto, que las estrategias institucionales para generar procesos de inclusión necesitan realizar ajustes en la política con modelos realmente inclusivos, que vengan a trasformar tanto los procesos administrativos como la formación de todos los protagonistas que interactúan con las diversas poblaciones que se incorporan a las instituciones de educación superior (Zapata, 2013).

Actualmente, ante los retos de la sociedad del conocimiento, se identifica una propuesta de gran relevancia e innovación en el ámbito educativo, el enfoque socioformativo, como un planteamiento inclusivo que potencia la formación integral de las personas a través del abordaje de problemas situados, con base en la colaboración y dirijidos a la trasformación o creación de nuevos conocimientos de acuerdo con la sociedad del conocimiento, enfoque que admite la incorporación de nuevas consideraciones epistemológicas (Escudero, 2018). En este tipo de sociedad, se demanda impulsar y fortalecer los procesos de una educación incluyente, una educación que contemple a todos, que identifique y valore la diversidad como oportunidad para el aprendizaje, siempre en un marco de respeto y tolerancia, en escenarios educativos en donde docentes y alumnos aprendan a convivir de manera colaborativa, con un proyecto ético de vida, enfocándose en el mejoramiento de las condiciones de vida a través de la gestión del conocimiento en fuentes confiables y por medio del abordaje de problemas contextuales (Tobón, 2017).

Una línea de estudio en la socioformación es contribuir a generar un marco teórico y metodológico de la inclusión que lleve a fortalecer el tejido social donde todos los ciudadanos posean los mismos derechos y trabajen juntos tanto en su realización como seres humanos como en la mejora de las condiciones de vida (de la Oliva, Tobón, Pérez, Romero, 2015). Sin duda, se hace necesaria una formación docente desde la socioformación, que permita contar con profesores comprometidos y responsables de su propia formación y actuación social. En este marco, la educación requiere de cambios sustantivos para dar respuesta a los retos de la sociedad del conocimiento, que den respuesta a la diversidad de alumnos y docentes, se establezcan políticas inclusivas, se formen culturas inclusivas $y$, sobre todo, se desarrollen prácticas inclusivas desde el enfoque de la socioformación (Tobón, 2018).

Como se menciona anteriormente, se han desarrollado investigaciones respecto a los procesos que requieren las escuelas para convertirse en un espacio inclusivo; en este aspecto, uno 
de los instrumentos que ha sido el principal referente para apoyar el proceso de desarrollo hacia escuelas inclusivas es el instrumento del Índice de Inclusión, impulsado por Ainscow y Booth como una contribución para ampliar el conocimiento acerca de la inclusión en todas sus manifestaciones (Ortega, Cilleros, Río, 2017), y tiene como propósito apoyar a las escuelas en el proceso de inclusión, ya que permite un proceso de autoevaluación que involucra a todos los actores, desde directivos, docentes, padres de familia, alumnos y otros miembros de la comunidad (García, 2015). Ante eso, en México, se han trabajado adaptaciones del Índice de Inclusión. Plancarte (2010) realizó una investigación cuyo propósito fue llevar a cabo la validación de constructo y contenido del Índice de Inclusión para su adaptación a las características socioculturales y educativas de la población mexicana; en el proceso encontró que existen desacuerdos en diferentes secciones e indicadores.

Algunos estudios realizados que exploran acerca de instrumentos que evalúan la inclusión en educación superior, dan cuenta de pocas investigaciones enfocadas a validar instrumentos, encuestas y cuestionarios que se utilizan para medir la inclusión en la educación en este nivel educativo; en los documentos referidos se encontró una coincidencia en relación con la necesidad de realizar este tipo de estudios; lo que permite que el tema de la evaluación de la inclusión es incipiente en la educación superior y que, de acuerdo a la revisión, no se cuenta con instrumentos validados y sometidos a procesos de confiabilidad para evaluar la inclusión en las escuelas normales. Diseñar instrumentos conceptualizados desde la socioformación, y que estos sean válidos y confiables, toma una importancia fundamental en la actualidad educativa (Ibarra, Segredo, Juárez, Tobón, 2018).

De esta manera, contar con instrumentos validados y sometidos a procesos de confiabilidad para diagnosticar el proceso de inclusión en las escuelas normales resultaría relevante, con el fin de propiciar la mejora de los procesos de formación en las instituciones formadoras de docentes e impulsar una cultura diferente para educar en la sociedad del conocimiento. El propósito del estudio consistió en someter a un proceso de validación y confiabilidad la rúbrica para evaluar procesos de inclusión en las escuelas normales, la cual fue diseñada y evaluada mediante juicio de expertos para determinar su validez de contenido previo a este estudio, señalando que su proceso de elaboración se sustentó en la metodología planteada por Hernández y Tobón (2016) para realizar la evaluación desde un enfoque socioformativo.

Considerando lo anterior, el presente estudio se enfocó en las siguientes metas: 1) ejecutar un análisis factorial para valorar sus dimensiones y contrastarlas con la teoría para obtener, mediante esta técnica, la validez de constructo del instrumento; y 2) analizar la confiabilidad del instrumento aplicándolo como mínimo a una muestra de 400 personas entre directivos, docentes y estudiantes de escuelas normales del país para realizar el cálculo del coeficiente Alfa de Cronbach. De esta manera, este podrá ser implementado de forma sistemática en las escuelas normales y, con ello, llevar a cabo acciones que fortalezcan sus procesos de mejora,

Diólo@os sobre Educación TEMAS ACTUALES EN INVESTIGACION : año 12 | número 22 | enero-junio 2021 | ISSN 2007-2171 
particularmente en lo referente a los procesos de inclusión. También será de utilidad en futuras investigaciones en torno al tema.

\section{Metodología}

\section{Tipo de estudio}

Se realizó un estudio instrumental de validez de constructo mediante el análisis factorial y de confiabilidad respecto al instrumento que evalúa los procesos de inclusión en la educación normal.

\section{Participantes}

Para realizar el estudio se aplicó el cuestionario a un total de 433 personas entre directivos, docentes y estudiantes de 11 escuelas normales en México.

Tabla 1. Total de personas de escuelas normales que aplicaron el cuestionario

\begin{tabular}{|l|c|}
\hline Función en la institución & Número de personas \\
\hline Directivos & 24 \\
\hline Docentes & 107 \\
Estudiantes & 302 \\
\hline Total de personas & 433 \\
\hline
\end{tabular}

Fuente: elaboración propia.

De este total, se presentan en las siguientes tablas los datos sociodemográficos de los participantes por el tipo de función que desempeñan en la escuela normal.

Tabla 2. Datos sociodemográficos de los participantes con función de directivos en la escuela normal

\begin{tabular}{|l|l|}
\hline Sexo & $54.16 \%$ hombres \\
\hline Promedio de edad & $45.84 \%$ mujeres \\
\hline Estado civil & 49.58 años \\
& $66.66 \%$ Casado \\
& $12.5 \%$ Unión libre \\
& $16.66 \%$ Soltero \\
& $4.18 \%$ Divorciado \\
\hline Nivel de escolaridad & $4.18 \%$ Postdoctorado \\
& $20.83 \%$ Doctorado \\
& $66.66 \%$ Maestría \\
& $8.33 \%$ Licenciatura \\
\hline
\end{tabular}

Fuente: adaptado de cuestionario sociodemográfico CIFE. 
Tabla 3. Datos sociodemográficos de los participantes con función de docente en la escuela normal

\begin{tabular}{|l|c|}
\hline Sexo & $38.68 \%$ hombres \\
& $61.32 \%$ mujeres \\
\hline Promedio de edad & 45.86 años \\
\hline Estado civil & $66.03 \%$ Casado \\
& $5.67 \%$ Unión libre \\
& $18.86 \%$ Soltero \\
& $7.55 \%$ Divorciado \\
& $1.89 \%$ Viudo \\
\hline Nivel de escolaridad & $0 \%$ Postdoctorado \\
& $13.21 \%$ Doctorado completo \\
& $8.50 \%$ Doctorado incompleto \\
& $59.41 \%$ Maestría completa \\
& $5.65 \%$ Maestría incompleta \\
& $13.21 \%$ Licenciatura \\
\hline
\end{tabular}

Fuente: adaptado de cuestionario sociodemográfico CIFE.

Tabla 4. Datos sociodemográficos de los participantes estudiantes de la escuela normal

\begin{tabular}{|l|c|}
\hline Sexo & $39.94 \%$ hombres \\
& $60.06 \%$ mujeres \\
\hline Promedio de edad & 20.50 años \\
\hline Estado civil & $1.65 \%$ Casado \\
& $1.65 \%$ Unión libre \\
& $96.70 \%$ Soltero \\
& $0 \%$ Divorciado \\
& $0 \%$ Viudo \\
\hline
\end{tabular}

Fuente: adaptado de cuestionario sociodemográfico CIFE.

Para la determinación de la muestra se empleó un procedimiento no probabilístico utilizando la técnica de conveniencia que, según Arias, Villasís y Novales (2016), consiste en la selección por métodos no aleatorios de una muestra cuyas características sean similares a las de la población objetivo, considerando el investigador seleccionar directa e intencionadamente los individuos de la población, como fue el caso del presente estudio, donde se determinó un número mínimo de personas a encuestar (400 personas entre directivos, docentes y estudiantes) en 11 escuelas normales del país, que se consideró cumplían con características que representan a la población objeto de estudio. Se solicitó a los cuerpos directivos de estas instituciones hicieran la petición al personal directivo, docente y estudiantes de últimos semestres (séptimo y octavo semestre) de los diferentes programas de licenciatura que en estas se ofrecen para la resolución de dicho cuestionario, el total de encuestas resueltas dependió principalmente de 
la disposición del personal de cada institución. Cabe precisar, para efectos de optimar recursos económicos y tiempos debido al tamaño de la población muestra, el cuestionario se alojó en una plataforma para crear encuestas en línea administrado por el Centro Universitario CIFE, de esta manera se envió el link a la encuesta al personal designado por la institución para que coordinara dicha aplicación.

\section{Procedimiento}

\section{- Análisis factorial}

Para el proceso de validación se realizó el análisis factorial exploratorio (EFA) de ítems con la misma muestra empleada para el estudio de confiabilidad, que es una de las técnicas más frecuentemente aplicadas en estudios relacionados con el desarrollo y validación de test, así también, es la técnica por excelencia utilizada para explorar el conjunto de variables latentes o factores comunes que explican las respuestas a los ítems de un test (Lloret, Ferreres, Hernández, Tomás, 2014). Antes de la aplicación del EFA, se realizaron una serie de procedimientos que, según Costello y Osborne (2005), sirven para determinar el cumplimiento de requisitos de esta técnica. Se obtuvo una matriz de correlaciones, se calculó el valor de la determinante de la matriz de correlaciones, el índice de KMO (Kaiser Meyer Olkin) y se realizó la prueba de esfericidad de Barlett.

\section{- Estudio de confiabilidad}

Se aplicó el instrumento a una muestra no probabilística de 433 personas entre directivos, docentes y estudiantes de 11 escuelas normales en México. Se utilizó el coeficiente de Alfa de Cronbach, ampliamente utilizado como una forma sencilla y confiable para la validación de constructo de una escala y como una medida que cuantifica la correlación existente entre los ítems que componen un instrumento (Alonso y Santacruz, 2015). El cálculo del coeficiente permite determinar la confiabilidad para asegurar que cualquier procedimiento de medición genere los mismos resultados en eventos repetidos, con el fin de obtener consistencia en los resultados, lo que determinará el nivel de confiabilidad del proceso (Mendoza, Garza, 2009).

\section{Aspectos éticos}

El presente estudio se apegó a la Ley de Protección de Datos Personales vigentes en México. En la encuesta en línea que se aplicó a los informantes de las escuelas normales seleccionadas se informó sobre los propósitos del estudio, solicitando en la misma encuesta su consentimiento, bajo la consideración del encuestado retirarse en cualquier momento del estudio. 


\section{Resultados}

En primer lugar, en la tabla 5 se presentan los datos descriptivos del instrumento "Rúbrica para diagnosticar los procesos de inclusión en las escuelas normales".

Tabla 5. Rúbrica para diagnosticar los procesos de inclusión en las escuelas normales

\begin{tabular}{|c|c|}
\hline Criterios & Niveles de dominio \\
\hline $\begin{array}{l}\text { ¿Qué acciones realiza el equipo líder en tu } \\
\text { institución para promover los procesos de } \\
\text { inclusión? }\end{array}$ & $\begin{array}{l}\text { 1. Receptivo (Insuficiente). El equipo líder comparte } \\
\text { información sobre la inclusión en la educación normal } \\
\text { para generar protocolos administrativos que la descri- } \\
\text { ban. } \\
\text { 2. Resolutivo (Suficiente). El equipo líder se enfoca en } \\
\text { dar respuesta a los requerimientos administrativos y } \\
\text { pedagógicos relacionados con la inclusión, mediante la } \\
\text { generación de procedimientos técnicos y curriculares } \\
\text { que se deben implementar a nivel institucional. } \\
\text { 3. Autónomo (Satisfactorio). El equipo líder analiza } \\
\text { el contexto institucional y social para establecer un } \\
\text { procedimiento de mejoramiento que garantice la im- } \\
\text { plementación de los procesos de inclusión de forma } \\
\text { colaborativa y participativa. } \\
\text { 4. Estratégico (Destacado). El equipo líder genera es- } \\
\text { trategias para la trasformación del entorno escolar, } \\
\text { familiar, comunitario y social, considerando los apor- } \\
\text { tes de la educación inclusiva. }\end{array}$ \\
\hline $\begin{array}{l}\text { ¿Qué características tienen las metas pro- } \\
\text { puestas para favorecer los procesos de inclu- } \\
\text { sión en tu institución? }\end{array}$ & $\begin{array}{l}\text { 1. Receptivo (Insuficiente). Las metas diseñadas con- } \\
\text { sideran los principios de la educación inclusiva a partir } \\
\text { de la propuesta de autores relevantes a nivel interna- } \\
\text { cional. } \\
\text { 2. Resolutivo (Suficiente). Las metas diseñadas con- } \\
\text { tienen aspectos técnicos y administrativos que res- } \\
\text { ponden a las necesidades del contexto institucional y } \\
\text { orientan hacia el cumplimiento de los requerimientos } \\
\text { establecidos por la autoridad educativa. } \\
\text { 3. Autónomo (Satisfactorio). Las metas diseñadas } \\
\text { retoman las necesidades detectadas por el equipo y } \\
\text { muestran indicadores de logro de manera organizada, } \\
\text { que van de lo general a lo particular. } \\
\text { 4. Estratégico (Destacado). Las metas diseñadas apor- } \\
\text { tan elementos innovadores y creativos para trasformar } \\
\text { el contexto institucional en un entorno inclusivo de } \\
\text { manera sistémica. }\end{array}$ \\
\hline
\end{tabular}




\begin{tabular}{|c|c|}
\hline $\begin{array}{l}\text { ¿Qué aspectos se consideraron en el diag- } \\
\text { nóstico institucional (interno y externo)? }\end{array}$ & $\begin{array}{l}\text { 1. Receptivo (Insuficiente). El diagnóstico interno } \\
\text { describe los procedimientos curriculares y adminis- } \\
\text { trativos, mientras que el externo hace una lista de las } \\
\text { características de la comunidad. } \\
\text { 2. Resolutivo (Suficiente). El diagnóstico interno des- } \\
\text { cribe las necesidades de los alumnos, profesores y } \\
\text { directivos, mientras que el externo analiza las proble- } \\
\text { máticas de la comunidad. } \\
\text { 3. Autónomo (Satisfactorio). El diagnóstico interno } \\
\text { integra referentes teóricos que permiten interpretar y } \\
\text { argumentar las necesidades de los alumnos, profeso- } \\
\text { res y directivos, mientras que el externo relaciona las } \\
\text { problemáticas de la comunidad con las dificultades de } \\
\text { aprendizaje en el aula. } \\
\text { 4. Estratégico (Destacado). El diagnóstico interno re- } \\
\text { laciona las necesidades curriculares y del aprendizaje } \\
\text { con posibles acciones de mejoramiento a nivel insti- } \\
\text { tucional, mientras que el externo establece acciones } \\
\text { de vinculación con la comunidad para responder a las } \\
\text { necesidades del entorno. }\end{array}$ \\
\hline $\begin{array}{l}\text { ¿En qué grado se atienden los problemas del } \\
\text { contexto educativo relacionados con los pro- } \\
\text { cesos de inclusión? }\end{array}$ & $\begin{array}{l}\text { 1. Receptivo (Insuficiente). Se identifican problemas } \\
\text { relacionados con las necesidades académicas y opera- } \\
\text { tivas de los profesores y alumnos. } \\
\text { 2. Resolutivo (Suficiente). Se abordan problemas re- } \\
\text { lacionados con las necesidades de aprendizaje de los } \\
\text { alumnos y del desempeño profesional del docente. } \\
\text { 3. Autónomo (Satisfactorio). Se analizan las necesi- } \\
\text { dades, vacíos o carencias de los profesores y alumnos } \\
\text { respecto al logro de las metas establecidas a nivel ins- } \\
\text { titucional. } \\
\text { 4. Estratégico (Destacado). Se proponen acciones de } \\
\text { mejoramiento y atención a las necesidades, vacíos y } \\
\text { carencias que manifiestan docentes y alumnos, con- } \\
\text { siderando acciones concretas, sistemáticas y de alto } \\
\text { impacto (trasformación). }\end{array}$ \\
\hline $\begin{array}{l}\text { ¿Qué elementos se consideran en las pla- } \\
\text { neaciones didácticas para el abordaje de los } \\
\text { procesos de inclusión? }\end{array}$ & $\begin{array}{l}\text { 1. Receptivo (Insuficiente). Las planeaciones didácticas } \\
\text { muestran las competencias y los contenidos corres- } \\
\text { pondientes a los programas o asignaturas. } \\
\text { 2. Resolutivo (Suficiente). Las planeaciones didácticas } \\
\text { integran estrategias para promover la inclusión y los } \\
\text { procesos de evaluación de estas. } \\
\text { 3. Autónomo (Satisfactorio). Las planeaciones didácti- } \\
\text { cas integran problemas del contexto de los estudian- } \\
\text { tes y docentes que favorecen la inclusión como parte } \\
\text { esencial de las prácticas educativas. } \\
\text { 4. Estratégico (Destacado). Las planeaciones didác- } \\
\text { ticas integran procesos de evaluación con base en } \\
\text { criterios y evidencias que abordan la inclusión como } \\
\text { propuesta metodológica a nivel aula. }\end{array}$ \\
\hline
\end{tabular}




\begin{tabular}{|c|c|}
\hline $\begin{array}{l}\text { ¿Qué aspectos didácticos se consideran en } \\
\text { los procesos de evaluación inclusivos? }\end{array}$ & $\begin{array}{l}\text { 1. Receptivo (Insuficiente). Se planean actividades cen- } \\
\text { tradas en la apropiación de conceptos, la organización } \\
\text { de información y el manejo de procedimientos. } \\
\text { 2. Resolutivo (Suficiente). Se planean actividades cen- } \\
\text { tradas en la resolución de situaciones que aportan un } \\
\text { sentido al manejo de los contenidos disciplinares, y } \\
\text { permiten generar productos de aprendizaje. } \\
\text { 3. Autónomo (Satisfactorio). Se planean actividades } \\
\text { de forma articulada, orientadas a la resolución de un } \\
\text { problema de contexto y la generación de productos } \\
\text { que demuestran el nivel de desempeño de los partici- } \\
\text { pantes. } \\
\text { 4. Estratégico (Destacado). Se planean actividades que } \\
\text { promueven la mejora del entorno y la resolución de } \\
\text { necesidades en el contexto escolar, personal, familiar, } \\
\text { profesional, etc. los procesos de evaluación permiten } \\
\text { identificar los logros y los aspectos a mejorar de mane- } \\
\text { ra colaborativa. }\end{array}$ \\
\hline $\begin{array}{l}\text { ¿Qué características tiene la retroalimenta- } \\
\text { ción de los procesos de inclusión educativa } \\
\text { en tu institución? }\end{array}$ & $\begin{array}{l}\text { 1. Receptivo (Insuficiente). La retroalimentación busca } \\
\text { mejorar los procesos administrativos y curriculares de } \\
\text { forma general. } \\
\text { 2. Resolutivo (Suficiente). La retroalimentación pro- } \\
\text { porciona elementos técnicos y pedagógicos para me- } \\
\text { jorar las prácticas educativas y administrativas. } \\
\text { 3. Autónomo (Satisfactorio). La retroalimentación per- } \\
\text { mite establecer acciones específicas para atender las } \\
\text { necesidades institucionales en todos los ámbitos. } \\
\text { 4. Estratégico (Destacado). La retroalimentación apor- } \\
\text { ta elementos innovadores y creativos para atender las } \\
\text { necesidades institucionales y del contexto social. }\end{array}$ \\
\hline
\end{tabular}

Fuente: elaboración propia.

Como se mencionó anteriormente, a partir del grupo en estudio se analizó la validez de constructo y la confiabilidad del instrumento. En primer lugar, se presentan los resultados del análisis factorial, en la tabla 6 se encontró que cumple los requisitos para el análisis factorial exploratorio: el valor de la determinante de la matriz de correlaciones fue de .012; la prueba de Bartlett fue de Xi: 1316.908 ( $p<0.001$ ) y el Índice KMO de .909, que de acuerdo con Lloret, Ferreres, Hernández y Tomás (2014) se considera satisfactorio cuando son valores de .80 en adelante. 
Tabla 6. Prueba de índice KMO y Bartlett

\begin{tabular}{|l|l|r|}
\hline Medida Kaiser-Meyer-Olkin de adecuación de muestreo & .909 \\
\hline Prueba de esfericidad de Bartlett & Aprox. Chi-cuadrado & 1316.908 \\
\cline { 2 - 3 } & gl & 21 \\
\cline { 2 - 3 } & Sig. & .000 \\
\hline
\end{tabular}

Así, también se obtuvieron correlaciones estadísticamente significativas entre los ítems de la rúbrica (tabla 7).

Tabla 7. Matriz de correlaciones ${ }^{\mathrm{a}}$

\begin{tabular}{|c|c|c|c|c|c|c|c|c|}
\hline & & $\begin{array}{c}\text { VARoo } \\
001\end{array}$ & $\begin{array}{c}\text { VARoo } \\
002\end{array}$ & $\begin{array}{c}\text { VARoo } \\
003\end{array}$ & $\begin{array}{c}\text { VARoo } \\
004\end{array}$ & $\begin{array}{c}\text { VARoo } \\
005\end{array}$ & $\begin{array}{c}\text { VARoo } \\
006\end{array}$ & $\begin{array}{c}\text { VAROo } \\
007\end{array}$ \\
\hline Correlación & VARoo001 & 1.000 & .689 & .606 & .556 & .523 & .576 & .550 \\
\hline & VARoooo2 & .689 & 1.000 & .582 & .552 & .524 & .549 & .568 \\
\hline & VARoooo3 & .606 & .582 & 1.000 & .645 & .576 & .623 & .572 \\
\hline & VARoo004 & .556 & .552 & .645 & 1.000 & .640 & .620 & .643 \\
\hline & VARoo005 & .523 & .524 & .576 & .640 & 1.000 & .720 & 697 \\
\hline & VARoooo6 & .576 & .549 & .623 & .620 & .720 & 1.000 & .725 \\
\hline & VARoooo7 & .550 & .568 & .572 & .643 & .697 & .725 & 1.000 \\
\hline Sig. (unila- & VARoo001 & & .000 & .000 & .000 & .000 & .000 & .000 \\
\hline & VARoooo2 & .000 & & .000 & .000 & .000 & .000 & .000 \\
\hline & VARoooo3 & .000 & .000 & & .000 & .000 & .000 & .000 \\
\hline & VARoo004 & .000 & .000 & .000 & & .000 & .000 & .000 \\
\hline & VARoo005 & .000 & .000 & .000 & .000 & & .000 & .000 \\
\hline & VARoooo6 & .000 & .000 & .000 & .000 & .000 & & .000 \\
\hline & VARoooo7 & .000 & .000 & .000 & .000 & .000 & .000 & \\
\hline
\end{tabular}

Nota: ${ }^{\text {a }}$ Determinante $=.012$.

De acuerdo con los resultados obtenidos, los valores de las comunalidades fueron adecuados y se representan en el modelo factorial mostrado en la tabla 8. El EFA extrajo un componente principal, afirmando con ello que existe un único factor sudyacente a todos los ítems que componen la rúbrica, componente que explica $60.767 \%$ de la varianza (tabla 9). Este factor presentó autovalores iniciales de 4.642 (tabla 9). 
Tabla 8. Comunalidades

\begin{tabular}{|l|c|c|}
\hline & Inicial & Extracción \\
\hline VARoo001 & .562 & .547 \\
\hline VARoo002 & .549 & .534 \\
\hline VARoo003 & .553 & .590 \\
\hline VARoo004 & .569 & .613 \\
\hline VARoo005 & .615 & .628 \\
\hline VARoo006 & .654 & .683 \\
\hline VARoo007 & .631 & .658 \\
\hline
\end{tabular}

Nota. Método de extracción: factorización de eje principal.

Tabla 9. Varianza total explicada

\begin{tabular}{|l|r|r|r|r|r|r|}
\hline & \multicolumn{4}{|c|}{ Autovalores iniciales } & \multicolumn{3}{c|}{$\begin{array}{c}\text { Sumas de extracción de cargas } \\
\text { al cuadrado }\end{array}$} \\
\cline { 2 - 7 } Factor & Total & $\begin{array}{c}\text { \% de } \\
\text { varianza }\end{array}$ & $\begin{array}{c}\text { \% } \\
\text { acumulado }\end{array}$ & Total & $\begin{array}{c}\text { \% } \\
\text { de varianza }\end{array}$ & $\begin{array}{c}\text { \% } \\
\text { acumulado }\end{array}$ \\
\hline 1 & 4.642 & 66.321 & 66.321 & 4.254 & 60.767 & 60.767 \\
\hline 2 & .681 & 9.733 & 76.054 & & & \\
\hline 3 & .457 & 6.531 & 82.585 & & & \\
\hline 4 & .364 & 5.194 & 87.779 & & & \\
\hline 5 & .310 & 4.434 & 92.213 & & & \\
\hline 6 & .298 & 4.257 & 96.470 & & & \\
\hline 7 & .247 & 3.530 & 100.000 & & & \\
\hline
\end{tabular}

Nota. Método de extracción: factorización de eje principal.

Tabla 10. Matriz factorial ${ }^{\mathrm{a}}$

\begin{tabular}{|l|c|}
\hline & Factor \\
\cline { 2 - 2 } & 1 \\
\hline VARoo001 & .740 \\
\hline VAR00002 & .731 \\
\hline VARoo003 & .768 \\
\hline VAR00004 & .783 \\
\hline VARoo005 & .793 \\
\hline VAR00006 & .826 \\
\hline VARoo007 & .811 \\
\hline
\end{tabular}

Nota. Método de extracción: factorización de eje principal.

a. 1 factores extraídos. 4 iteraciones necesarias. 
Finalmente, en la tabla 11 se presentan los datos de confiabilidad a partir de la aplicación del instrumento a la muestra participante. Puede observarse que el instrumento presenta alta confiabilidad dado que el Alfa de Cronbach fue de 0.915 con $\mathrm{p}<0.05$.

Tabla 11. Estadísticas de confiabilidad

\begin{tabular}{|c|c|c|}
\hline Alfa de Cronbach & $\begin{array}{l}\text { Alfa de Cronbach basada en } \\
\text { elementos estandarizados }\end{array}$ & No. de elementos \\
\hline .915 & .915 & 7 \\
\hline
\end{tabular}

\section{Discusión}

A partir del estudio realizado, puede concluirse que el instrumento es pertinente para evaluar y diagnosticar los procesos de inclusión en las escuelas normales, tomando como base el estudio de análisis factorial llevado a cabo para determinar la validez de constructo del instrumento. La técnica utilizada fue el análisis factorial exploratorio (EFA), que consiste en un conjunto de métodos estadísticos multivariados de interdependencia cuyo propósito principal es identificar una estructura de factores subyacentes a un conjunto amplio de datos (Pérez y Medrano, 2010). Diversos estudios señalan la importancia del análisis factorial para asegurar la pertinencia de los instrumentos, en razón de que con mucha frecuencia se diseñan instrumentos con bajo grado de coherencia con la teoría y el entorno. Ferrando y Anguiano (2010) señalan que la mayor parte de los estudios factoriales en ramas de las ciencias sociales utilizan este tipo de análisis para evaluar la estructura de una prueba a partir de las puntuaciones en sus ítems, o hipótesis de tipo dimensional utilizando como medidas puntuaciones en diferentes tests. Asimismo, el análisis factorial exploratorio de ítems, es una de las técnicas más comúnmente aplicadas en estudios relacionados con el desarrollo y la validación de pruebas, ya que es la técnica por excelencia utilizada en la exploración del conjunto de variables latentes o factores comunes que explican las respuestas a los ítems de una prueba (Lloret, Ferreres, Hernández, Tomás, 2014).

En este sentido, los resultados de la validez de constructo mostraron la predominancia de un factor, en el cual se incluyen los procesos de inclusión en las escuelas normales, afirmando con ello que existe un único factor subyacente a todos los ítems que componen la rúbrica. Esto está acorde con lo que plantean Pérez y Medrano (2010), la aproximación al constructo u objeto de estudio permite identificar, a partir de las relaciones entre las variables observables, la existencia de factores subyacentes de considerable valor teórico, y que se deben considerar las limitaciones del AFE, cuyas consecuencias son que su uso exclusivo supone una aproximación débil a la validación de un constructo. Sin embargo, en el presente estudio se realizó el análisis exploratorio, encontrándose que sí posee una concordancia significativa en su contenido, pertinencia y un alto nivel de confiabilidad. 
Soriano (2015) sostiene que la confiabilidad de un instrumento se refiere al grado en que un instrumento construido por varios ítems presenta una alta correlación y miden consistentemente una muestra. Es decir, la dimensión considerada para el diseño del instrumento. El estudio muestra que el instrumento posee valores altos de confiabilidad, tomando como referencia a Mendoza y Garza (2017), quienes establecen que valores de Alfa de Cronbach superiores a 0.90 indican alta confiabilidad, lo que significa un buen grado de consistencia interna en el instrumento. De esta manera, se puede plantear que el instrumento está acorde con los avances teóricos y puede ser relevante en futuras investigaciones.

Si tomamos en consideración que el presente estudio es de tipo exploratorio, es pertinente señalar que se deben llevar a cabo estudios posteriores para confirmar la información que hasta aquí arrojó el estudio, realizando de nueva cuenta la validez de constructo que busque un mejor control de la aplicación en los participantes para evitar que los usuarios se sientan presionados a responder con una determinada orientación, así como determinarse otros tipos de validez (Tobón, Herrera, Juárez, Hernández, 2018). Asimismo, se considera necesario implementar estudios mediante la aplicación del instrumento en otros contextos de la educación normal del país y, con las adaptaciones necesarias, en instituciones de educación superior que ofrecen programas educativos para la formación de profesores, que permitan validarlo en mejor grado.

\section{Referencias}

Arias-Gómez, J.; M. Á. Villasís-Keever; M. G. M. Novales (2016). El protocolo de investigación III: la población de estudio. Revista Alergia México, 63(2), 201-206. https://www.redalyc.org/ pdf/4867/486755023011.pdf

Costello, A.B.; J. W. Osborne (2005). Best Practices in Exploratory Factor Analysis: Four re-commendations for Getting the Most From your Analysis. Practical Assessment, Research \& Evaluation, 10(7), 1-9. https://www.researchgate.net/profile/David Morgan 19/post/ How can one help people create simple scales from Likertscored items/attachment/59d63b58c49f478072ea72f2/AS:273740917608454@1442276329184/download/ Costello+\%26+Osborne+2014.pdf

De la Oliva, D.; S. Tobón; A. K. Pérez; J. Romero (2015). El proceso de inclusión social desde la socioformación: Análisis de concepciones sobre discapacidad y necesidades educativas especiales. Revista Paradigma, 36(2), 49-73. http://revistas.upel.edu.ve/index.php/paradigma/article/view/3015

Durán Gisbert, D.; G. G. Climent (2017). La formación del profesorado para la educación inclusiva: Un proceso de desarrollo profesional y de mejora de los centros para atender la diversidad. http://www.repositoriocdpd.net:8080/bitstream/handle/123456789/1913/Art DuranGisbertD Formaciondelprofesorado.pdf?sequence $=1$ 
Escudero, A. (2018, junio). Consideraciones epistemológicas para el estudio de la Sociedad del Conocimiento. En Juárez-Hernández, L. G.; S. Tobón (coords.). Cuarto Congreso Internacional en Socioformación y Sociedad del Conocimiento. México: CIFE.

Ferrando, P. J.; C. Anguiano-Carrasco (2010). El análisis factorial como técnica de investigación en psicología. Papeles del Psicólogo, 31(1). https://www.redalyc.org/html/778/77812441003/

García, G. (2015). Escala de prácticas inclusivas en educación básica: confiabilidad y validez en una muestra mexicana. Revista Latinoamericana de Educación Inclusiva, 9(1), 77-93. https:// dialnet.unirioja.es/descarga/articulo/5155491.pdf

González Alonso, J.; M. Pazmiño Santacruz (2015). Cálculo e interpretación del Alfa de Cronbach para el caso de validación de la consistencia interna de un cuestionario, con dos posibles escalas tipo Likert. Revista Publicando, 2(1), 62-67. https://www.ssoar.info/ssoar/bitstream/ handle/document/42382/ssoar-revpublicando-2015-1-gonzalez alonso jorge et alCalculo e interpretacion del.pdf?sequence $=1$

Hernández-Ayala, H.; S. Tobón-Tobón (2016). Análisis documental del proceso de inclusión en la educación. Ra-Ximhai, 12(6), 399-420. file:///C:/Users/direccion/Downloads/AN\%C3\%81LISIS\%20DOCUMENTAL\%20DEL\%20PROCESO\%20DE\%20 INCLUSI\%C3\%93N\%20EN\%20LA\%20EDUCACl\%C3\%93N.pdf

Ibarra-Piza, S.; S. Segredo-Santamaria; L. G. Juarez-Hernandez; S. Tobon (2018). Estudio de validez de contenido y confiabilidad de un instrumento para evaluar la metodología socioformativa en el diseño de cursos. Revista Espacios 39(53). http://www.revistaespacios.com/ cited2017/cited2017-24.html

Latorre, L. V. (2013). La educación inclusiva en docentes en formación: su evaluación a partir de la teoría de facetas. Folios, (37). http://revistas.pedagogica.edu.co/index.php/RF/article/ view/1823/1795

Lloret-Segura, S.; A. Ferreres-Traver; A. Hernández-Baeza; I. Tomás-Marco (2014). El análisis factorial exploratorio de los ítems: una guía práctica, revisada y actualizada. Anales de Psicología / Annals of Psychology, 30(3), 1151-1169. http://revistas.um.es/analesps/article/download/ analesps.30.3.199361/165441

Mendoza, J.; J. B. Garza (2009). La medición en el proceso de investigación científica: Evaluación de validez de contenido y confiabilidad (Measurement in the scientific research process: Content validity and reliability evaluation). https://core.ac.uk/download/pdf/84812900.pdf

Ortega, M. G.; M. V. M. Cilleros; C. J. Río (2017). El Index para la inclusión: presencia, aprendizaje y participación. Revista de Educación Inclusiva, 7(3). http://revistaeducacioninclusiva.es/index.php/REl/article/view/139/133

Pérez, E. R.; L. A. Medrano (2010). Análisis factorial exploratorio: bases conceptuales y metodológicas. Revista Argentina de Ciencias del Comportamiento (RACC), 2(1), 58-66. https://dialnet. unirioja.es/descarga/articulo/3161108.pdf 
Plancarte, C. P. (2010). El Índice de inclusión como herramienta para la mejora escolar. Revista Iberoamericana de Educación, 145-166. https://rieoei.org/historico/documentos/rie54a07. pdf

SEP (2011). Acuerdo 592, por el que se establece la articulación de la Educación Básica. México: SEP. SEP (2012). Acuerdo 649 por el que se establece el Plan de Estudios para la Formación de Maestros de Educación Primaria Intercultural Bilingüe. http://www.dgespe.sep.gob.mx/public/normatividad/acuerdos/acuerdo 649.pdf

Soriano Rodríguez, A. M. (2015). Diseño y validación de instrumentos de medición. http://redicces.org.sv/jspui/bitstream/10972/2105/1/2\%20disenoyvalidacion dialogos $14 . p d f$

Tobón, S. (2017). Ejes esenciales de la sociedad del conocimiento y la socioformación. https:// cife.edu.mx/recursos/2018/09/06/ejes-esenciales-de-la-sociedad-del-conocimiento-y-lasocioformacion/

Tobón, S.; J. H. Pimienta-Prieto; S. R. Herrera-Meza; L. G. Juárez-Hernández; J. S. Hernández-Mosqueda (2018). Validez y confiabilidad de una rúbrica para evaluar las prácticas pedagógicas en docentes de Educación Media (SOCME-10). Revista Espacios, 39(53). https://www.researchgate. net/profile/Sergio Tobon4/publication/329161856 Validez y confiabilidad de una rubrica para evaluar las practicas pedagogicas en docentes de Educacion Media SOCME10 Validity and Reliability of a Rubric for High School Teachers\%27 Pedagogical Practic/links/5bf94518458515a69e386185/Validez-y-confiabilidad-de-una-rubricapara-evaluar-las-practicas-pedagogicas-en-docentes-de-Educacion-Media-SOCME-10-Validity-and-Reliability-of-a-Rubric-for-High-School-Teachers-Pedagogical-Practice.pdf

Zapata, S. A. (2013). Reflexiones sobre inclusión y educación superior. Revista de Investigaciones UNAD, 12(2), 57-67. http://hemeroteca.unad.edu.co/index.php/revista-de-investigacionesunad/article/viewFile/1176/1387 